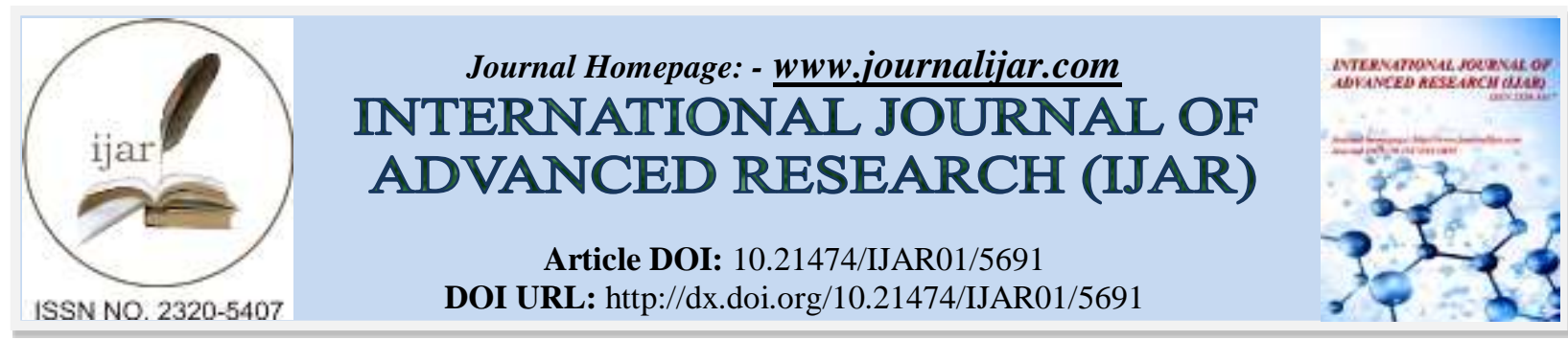

RESEARCH ARTICLE

\title{
THE EFFECT OF DIFFERENT OVARIAN STIMULATION PROTOCOLS ON VASCULAR ENDOTHELIAL GROWTH FACTOR (VEGF) CONCENTRATION IN THE SERUM AND FOLLICULAR FLUID OF WOMEN WITH PCOS UNDERGOING ICSI/IVF.
}

Nada Flayyih Hasan AL-Aboudy, Ula Mohammed Reda AlKawaz and Nawal Khairy Hussain AL- Ani. High Institute of Infersatility Diagnosis and Assisted Reproductive Technology Al-Nahrain University,Baghdad /Iraq.

\section{Manuscript Info}

Manuscript History

Received: 22 August 2017

Final Accepted: 24 September 2017

Published: October 2017

Key words:-

Vegf, pcos, ivf protocols.

\section{Abstract}

Objective: To measure the concentration of vascular endothelial growth factor (VEGF) in serum and follicular fluid of patients with PCOS at day of ova pick up after using of different IVF/ICSI protocols and to assess the pregnancy outcome.

Study design: A total of 90 infertile women (55 women with Polycystic ovary syndrome and the other 35 women as a control (non PCOS) undergoing controlled ovarian hyperstimulation for intracytoplasmic sperm injection cycle were prospectively recruited for this study in High Institute of Infertility Diagnosis and Assisted Reproductive Technology / AL-Nahrian University and Kamal ALSamarai Hospital, center of fertility and IVF (Baghdad/Iraq) during the period from December 2015 to the end of April 2017.The fifty five PCOS women were divided into three groups: twenty five (25) infertile women have had PCOS undergoing long Agonist protocol for ICSI cycle, fifteen (15) infertile women have had PCOS undergoing Antagonist protocol for ICSI and fifteen (15) infertile women have had PCOS undergoing short Agonist protocol for ICSI. The controlled group included 35 women were free of signs and symptoms of PCOS with regular cycles and no endocrine abnormalities undergoing ICSI cycle (15 infertile women underwent long agonist protocol, 10 women underwent short agonist and 10 women underwent antagonist protocol). In all patients, serum and follicular fluid VEGF levels were measured on the day of oocyte retrieval by using Enzyme linked immuno sorbent assay.

Results: There were, no significant difference in either serum or follicular fluid level of VEGF at the day of oocyte retrieval between the PCOS group and the non-PCOS group $(\mathrm{P}>0.05)$, no significant difference in the effect of different IVF/ICSI protocols (Long agonist, Antagonist, Short agonist) in the level of follicular \& serum VEGF of patients with PCOS $(\mathrm{P}>0.05)$ and no significant difference $(\mathrm{P}>0.05)$ in level of serum VEGF between pregnant \& non pregnant PCOS patients while shows significant difference $(\mathrm{P}<0.05)$ in level of follicular VEGF between pregnant \& non-pregnant PCOS patients. The level of follicular VEGF high in non-pregnant group in comparison with pregnant group. 
Conclusion: This study conclude, there was high level of follicular VEGF in non-pregnant PCOS patients at the day of oocyte retrieval in comparison with pregnant PCOS patients while no significant difference in level of serum VEGF between pregnant \&non pregnant PCOS patients, no significant difference in either serum or follicular fluid level of VEGF between the PCOS group and the non-PCOS group and no significant difference in the effect of different IVF/ICSI protocols in the level of follicular \& serum VEGF of patients with PCOS.

Copy Right, IJAR, 2017,. All rights reserved.

\section{Introduction:-}

Polycystic ovary syndrome (PCOS) is one of the most common endocrinopathies and metabolic abnormalities, affect 10-15\% of women of reproductive age was initially described by Stein and Leventhal in 1935, characterized by ovulatory dysfunction, biochemical and/or clinical hyperandrogenism including hirsutism, alopecia, obesity and polycystic ovarian morphology including the presence of hyper vascularized androgen secreting stroma and enlarged ovaries with multiple small follicles $2-8 \mathrm{~mm}_{\text {in }}$ diameter ${ }^{(1)}$. It may have complex effects on the ovulation, oocyte quality and the endometrium contributing to a lower fertility potential ${ }^{(2)}$. Fifty to seventy percent of women with PCOS are frequently associated with insulin resistance accompanied by compensatory hyperinsulinaemia and obesity. Insulin resistance is thought to play an important role in the aetiology of PCOS ${ }^{(3)}$.

VEGF is the prototypical member of a family of angiogenic factors which include placental growth factor, angiopoetin, basic fibroblast growth factor and VEGF A, B, C, D and E ${ }^{(4)}$. VEGF is expressed in many types of tissues and is upregulated during development, tissue remodeling, wound healing, and in several human disease states. In ovaries and the uterine endometrium, VEGF is expressed in granulosa and theca cells. Its regulate physiological and pathological angiogenesis which causes an increase in vascular permeability that mediated after administration of luteinizing hormone (LH) and human chorionic gonadotrophin(HCG) during ovarian stimulation cycles and have been implicated as a major factor in the pathophysiology of OHSS as high concentrations of VEGF have been found in ascites from patients with OHSS ${ }^{(5,6)}$. VEGF involved in the proliferation of capillaries that accompanies the selection of the preovulatory follicle resulting in an increased supply of nutrients and precursors, and therefore supporting the growth of the dominant follicle and its action required for successful blastocyst implantation in the rhesus monkey ${ }^{(7)}$ and also thought to be important for placental development ${ }^{(8)}$.

Serum VEGF increase in the luteal phase of the menstrual cycle and VEGF is suppressed by down-regulation of the reproductive axis. Abnormalities in ovarian angiogenesis may contribute not only to OHSS as seen in women with PCOS but also to disorders of ovulation, subfertility and pathogenic conditions such as endometriosis ${ }^{(9)}$. An increased expression of VEGF in the dense hyperthecotic stroma of polycystic ovaries which is associated with increased ovarian stromal blood flow could be the underlying cause of the loss of intra-ovarian autoregulatory mechanism seen in women with PCOS ${ }^{(10)}$. Recent studies have revealed that high levels of VEGF in serum and follicular fluid of PCOS patients plays a major role in follicular maturation and oocyte quality, they had fewer oocytes retrieved during the ART cycle, lower peak serum estradiol (E2) concentrations, consequently it has an impact on fertilization success and embryo development and reduced pregnancy rates in PCOS patients ${ }^{(11,12)}$.

\section{Subject, Material and methods:-}

A prospective case control study conducted in High Institute of Infertility Diagnosis and Assisted Reproductive Technology / AL-Nahrian University and Kamal AL-Samarai Hospital, center of fertility and IVF (Baghdad/Iraq) during the period from December 2015 to the end of April 2017.Ninety infertile couples (PCOS and controlled) have been enrolled in this study and enter their ICSI cycle. Informed consent of all patients was taken before inclusion in the study. All infertile couples were subjected to a full history taking, complete general and gynecological examination and full infertility investigations including: husband's seminal fluid analysis, hormonal assay, trans-vaginal ultrasound and hystrosalpingography for evaluation of uterine cavity and tubal patency and/or laproscopy for evaluation of tubal patency and exclusion of pelvic pathology. Fifty five women (55) with PCOS were included selected from those who undergoing $\mathrm{COH}$ for ICSI cycle. These fifty five women were divided into three groups: I. twenty five (25) infertile women have had PCOS undergoing long Agonist protocol for ICSI cycle. II. Fifteen (15) infertile women have had PCOS undergoing Antagonist protocol for ICSI. III. Fifteen (15) infertile 
women have had PCOS undergoing short Agonist protocol for ICSI. The diagnosis of PCOS depend on fulfilling at least two of three criteria based on the Rotterdam ESHRE/ASRMS sponsored PCOS consensus workshop group ${ }^{(13)}$. Rotterdam's criteria of PCOS which was based on: typical picture of polycystic ovaries on ultrasonography (ten or more follicles in each ovary, each follicle measuring 2-9 $\mathrm{mm}$ in diameter). 2- Anovulation or Oligo-ovulation. 3Hyperandrogenism; clinical or biochemical. Clinically hyperandrogenism manifested as a hirsutism, acne and biochemically as elevated serum testosterone level.

The controlled group included 35 women were free of signs and symptoms of PCOS with regular cycles and no endocrine abnormalities undergoing ICSI cycle (15 infertile women underwent long agonist protocol, 10 women underwent short agonist and 10 women underwent antagonist protocol) (13 with male factor infertility, 10 with unexplained infertility, and 12 with tubal infertility). The average age of women enrolled in this study ranged between 17 and 40 years had primary infertility (78 infertile couples) and secondary infertility (12 infertile couples) with duration between 2 and 15 years.

First group of PCOS patients (25) were enrolled in long protocol type of IVF/ICSI cycle, an ultrasound examination was performed in order to exclude those women with ovarian cyst and assess the endometrial thickness and started on day 21 of the previous menstrual cycle(mid-luteal) with a daily administration of subcutaneous injection of GnRH-a, triptorelin (Decapeptyl®; $0.1 \mathrm{mg}$ Ferring Co, Kiel, Germany) ${ }^{\circledR}$ for pituitary down-regulation and desensitization and continue till the day of HCG administration. When pituitary down-regulation was achieved (menstruation occur, E2 level reaching $<50 \mathrm{pg} / \mathrm{ml}$ and endometrial thickness was $\leq 2-3 \mathrm{~mm}$ on ultrasound examination ${ }^{(14)}$ ovarain stimulation started with recombinant human follicle stimulating hormone (rhFSH) (Gonal F, Merck Serono ${ }^{\circledR} 75$ IU of FSH activity per ampoules) by daily subcutaneous injection in a dose of 150-225 IU depending on the women's age and previous response of ovulation induction. The follicle growth and the doses of Gonal-F® were monitored by trans-vaginal ultrasound (cycle day 5 and subsequent scan were done every 2-3 days as required) and by serum E2 level (day 6-8 of (Gonal-F®) injection and till the day of hCG administration) ${ }^{(15)}$. When either two or three lead follicles have reached $17-18 \mathrm{~mm}$ ovulation induction was induced by the administration of recombinant hCG (rhCG 6500 IU, Ovitrelle®; Merck Serono, Italy) subcutaneously ${ }^{(16)}$. Second group (15) infertile couples in whom PCOS women were undergoing antagonist protocol, started daily administration of 150-225 IU of (Gonal-F®) injection subcutaneously from day two of menstrual cycle. GnRH antagonist (Cetrorelix) is usually given in a dose of $0.25 \mathrm{mg}$ daily when the leading follicle reaches a certain size by ultrasound monitoring $(12-14 \mathrm{~mm})$. The antagonist is continued together with the (Gonal-F®) stimulation until an adequate response is obtained and hCG injection requird for ovulation induction ${ }^{(17)}$. Third group (15) infertile couples in whom PCOS women were undergoing short agonist protocol, which started on day two of menstrual cycle with a daily administration of subcutaneous injection of GnRH-a, triptorelin (Decapeptyl®; $0.1 \mathrm{mg}$ Ferring Co, Kiel, Germany) ® .ovarain stimulation started on day three of menstrual cycle with daily administration of 150$225 \mathrm{IU}$ of (Gonal-F®) injection subcutaneously. Decapeptyl® is continued together with the (Gonal-F®) stimulation until an adequate response is obtained and $\mathrm{hCG}$ injection requird for ovulation induction ${ }^{(18)}$. The fourth controlled group contain thirty five infertile couples (35) in whom(15 undergoing long agonist protocol,10 undergoing short protocol,10 undergoing antagonist protocol). The oocytes were retrieved by transvaginal ultrasound-guided follicle aspiration 34-36 h after hCG administration. Sperm preparation and IVF/ICSI were performed and embryo transfers were carried out 2- 3 days after oocyte retrieval. Luteal phase support was done by giving $400 \mathrm{mg} / \mathrm{bid}$ of vaginal progesterone (Cyclogest ${ }^{\circledR}$, Actavis).A 5-ml blood sample was collected from all patients on the day of oocyte retrieval. After collection each sample was immediately centrifuged at $3000 \mathrm{rpm}$ for $10 \mathrm{~min}$ at room temperature and the supernatant was separated and stored at $-20{ }^{\circ} \mathrm{C}$ until assayed. A $2 \mathrm{ml}$ of clear Follicular fluid was collected from aspirated follicles, and then it was centrifuged at $3000 \mathrm{rpm}$ for $10 \mathrm{~min}$ at room temperature and stored at $-20{ }^{\circ} \mathrm{C}$ until assayed. VEGF levels were detected in both serum and follicular fluid samples by enzyme-linked immunosorbent assay technique using diagnostic kit (SHANGHAI YHB3181HU, China).

\section{Statistical analysis:-}

The Statistical Analysis System- SAS (2012), version 9 was used to evaluate effect of different factors in study parameters. Numeric variables were expressed as mean \pm standard error (SE). Least significant difference $-\mathrm{LSD}$ test (ANOVA) and T test were used to significant compare between means ${ }^{(19)}$. 


\section{Results:-}

Clinical characteristics of PCOS and control (according to certain parameters):

Female age: The mean age \pm standard error (SE) of all females with PCOS participate in this study was $(29.01 \pm 0.63)$ years, which is ranging between 17 and 40 years, and for control (the mean age \pm SE was (28.57 \pm 0.81$)$ years which ranging between 19 and 36 years. The statistical analysis showed no significant difference ( $>0.05)$ in the age between two groups (Table 1).

Body Mass Index (BMI): The mean \pm SE of BMI of PCOS patients in the present study was $(28.68 \pm 1.06) \mathrm{Kg} / \mathrm{m} 2$, and in control group the mean $\pm \mathrm{SE}$ of BMI was $(28.29 \pm 0.99) \mathrm{Kg} / \mathrm{m} 2$, (Table 1). The statistical analysis showed no significant difference ( $>0.05$ ) in the BMI between PCOS and control groups.

Duration of Infertility: The mean \pm SE regarding duration of infertility for all PCOS patients in the present study was $(6.67 \pm 0.37)$ years, and in control group was $(6.80 \pm 0.44)$ years. The statistical analysis showed no significant difference $(p>0.05)$ in the duration of infertility between two groups (Table 1$)$.

Table 1:- Clinical Characteristics of PCOS patients and non-PCOS groups.

\begin{tabular}{|c|c|c|c|c|c|}
\hline \multirow{2}{*}{ Characters } & \multicolumn{2}{|c|}{ PCOS } & \multicolumn{2}{c|}{ Non-PCOS } & \multirow{2}{*}{ LSD } \\
\cline { 2 - 5 } & Mean & SE & Mean & SE & \\
\hline Age(years) & 29.10 & 0.63 & 28.57 & 0.81 & $0.492 \mathrm{NS}$ \\
\hline BMI (kg/m2) & 28.68 & 1.06 & 28.29 & 0.99 & $0.562 \mathrm{NS}$ \\
\hline $\begin{array}{l}\text { Duration of } \\
\text { infertility }\end{array}$ & 6.67 & 0.37 & 6.80 & 0.44 & $0.465 \mathrm{NS}$ \\
\hline
\end{tabular}

All values are expressed as mean \pm SE (Standard Error).PCOS= poly cystic ovary syndrome; BMI=Body Mass Index; NS: Non-significant.

Comparison between PCO and Non-PCOS in ICSI Parameters:-

The Total Number of Oocytes Retrieved: The mean total number of oocytes \pm SE in both PCOS and non-PCOS group were (11.02 \pm 0.61 and $9.08 \pm 0.83)$ respectively. Total number of oocytes retrieved was significantly higher $(\mathrm{P}<0.05)$ in PCOS than that in non-PCOS (table 2$)$.

Number of mature Oocytes (Metaphase II): In this study, the mean number of mature oocytes [metaphase II (MII)] \pm SE in both PCOS and non-PCOS group were $(6.89 \pm 0.37$ and $5.05 \pm 0.46)$ respectively. The statistical analysis showed a highly significant difference $(\mathrm{P}<0.001)$ among the two groups (Table 2$)$.

Fertilization Rate (FR \%): Our study found that the mean number of fertilization rate \pm SE in both PCOS and nonPCOS group $(57.76 \pm 3.56$ and $55.16 \pm 6.08)$ respectively. There was no significant difference $(\mathrm{P}>0.05)$ between the two groups (Table 2).

Table 2:- Comparison between PCO and Non-PCOS in ICSI Parameters

\begin{tabular}{|c|c|c|c|c|}
\hline \multirow{2}{*}{ Group } & \multirow{2}{*}{ No. } & \multicolumn{3}{|c|}{ Mean \pm SE } \\
\cline { 3 - 5 } & & No. of Oocyte & No. of metaphase II(MII) & $\begin{array}{l}\text { Fertilization } \\
\text { Rate FR\% }\end{array}$ \\
\hline Non-PCO & 35 & $9.08 \pm 0.83$ & $5.05 \pm 0.46$ & $55.16 \pm 6.08$ \\
\hline PCO & 55 & $11.02 \pm 0.61$ & $6.89 \pm 0.37$ & $57.76 \pm 3.56$ \\
\hline P-value & --- & $0.051^{*}$ & $0.0029^{* *}$ & 0.695 NS \\
\hline \multicolumn{4}{|r|}{$*(\mathrm{P}<0.05), * *(\mathrm{P}<0.001)}$. \\
\hline
\end{tabular}

Comparison between PCOS patients and Non-PCOS in the level of Follicular Fluid \& Serum VEGF who underwent IVF/ICSI protocols:-

The mean of follicular and serum VEGF levels at the day of oocyte retrieval \pm SE of all the PCOS patients shared in this study were $(1519.09 \pm 81.22$ and $1678.95 \pm 100.50)$ respectively. The mean of follicular and serum VEGF levels at the day of oocyte retrieval \pm SE of all the non-PCOS group shared in this study were $(1512.02 \pm 110.17$ and 
$1548.46 \pm 80.95)$ respectively. The statistical analysis showed no significant difference $(\mathrm{P}>0.05)$ in level of follicular or serum VEGF between the two groups (Figure 1\&2).

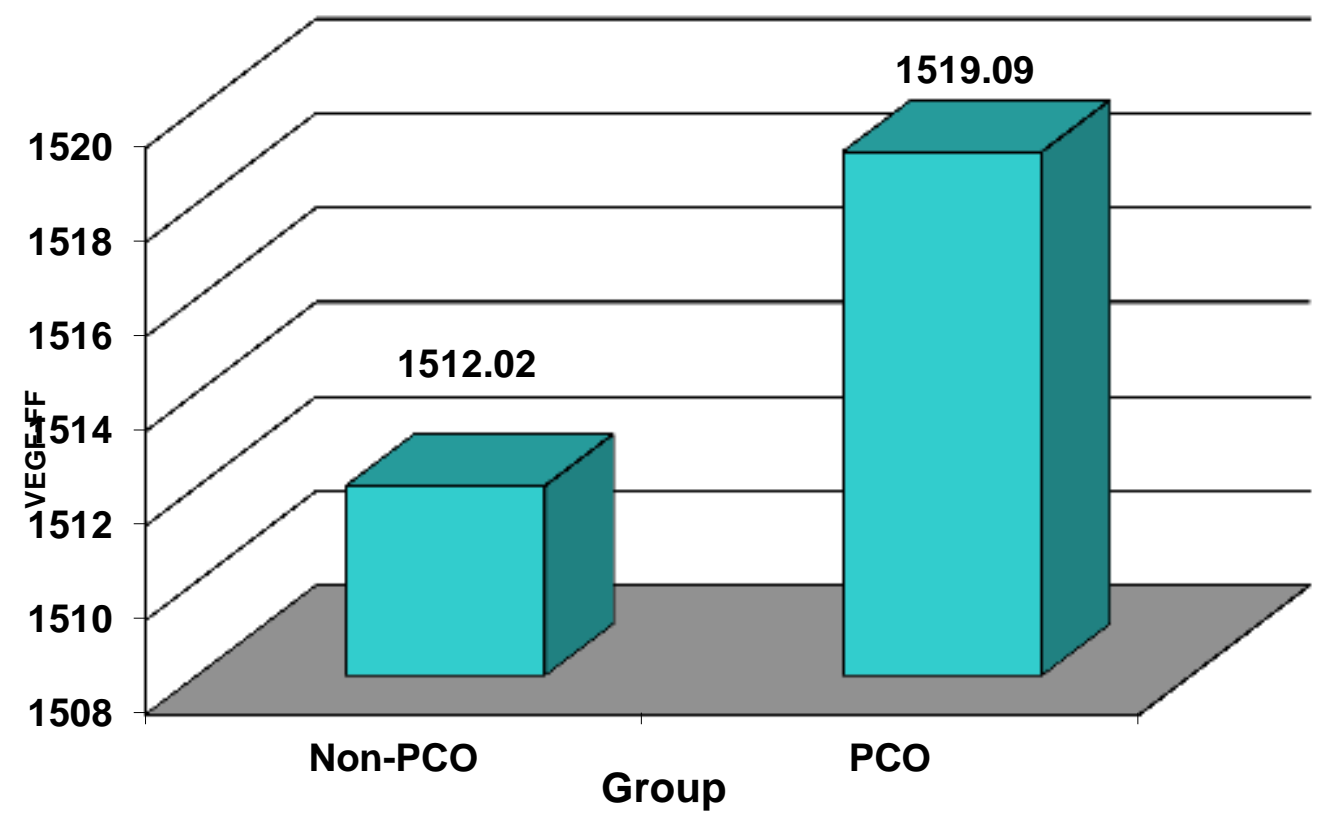

Figure 1. Comparison between PCO and Non-PCO in VEGF-FF

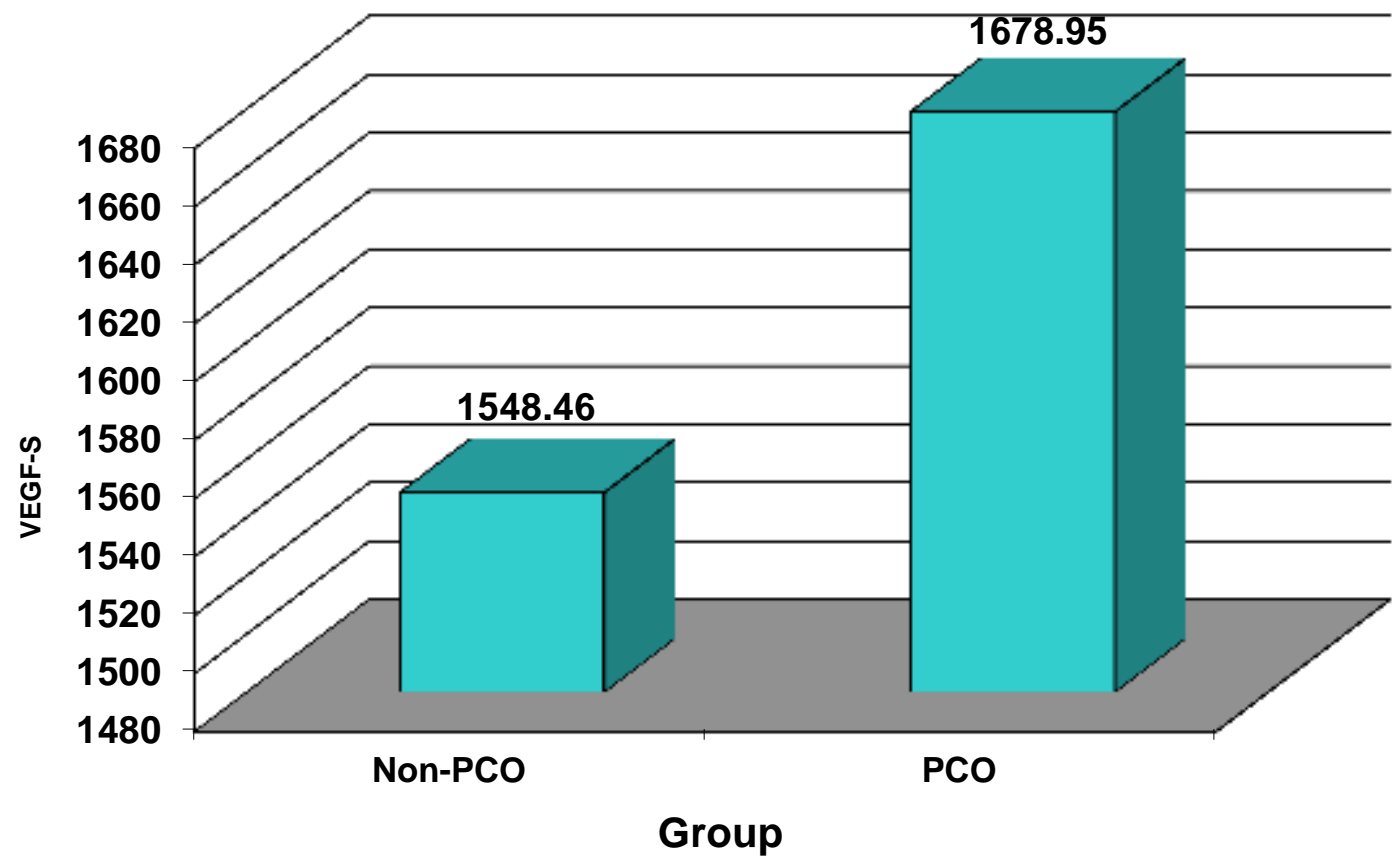

Figure 2. Comparison between PCO and Non-PCO in VEGF-S 
Comparison between the Effect of Different IVF/ICSI Protocols (Long agonist, Antagonist, Short agonist) in the level of Follicular \& Serum VEGF of patients with PCOS:-

The mean of follicular VEGF levels \pm SE of PCOS patients who underwent long agonist, Antagonist, short agonist protocols shared in this study were $(1527.91 \pm 164.03,1556.31 \pm 110.47$ and $1467.18 \pm 62.69)$ respectively. The mean of serum VEGF levels at the day of oocyte retrieval \pm SE of PCOS patients who underwent long agonist, Antagonist, short agonist protocols shared in this study were $(1650.96 \pm 162.65,1854.85 \pm 245.81$ and $1549.71 \pm$ $57.85)$ respectively. The statistical analysis showed no significant difference $(\mathrm{P}>0.05)$ in level of follicular or serum VEGF between the three groups (Figure 3, 4).

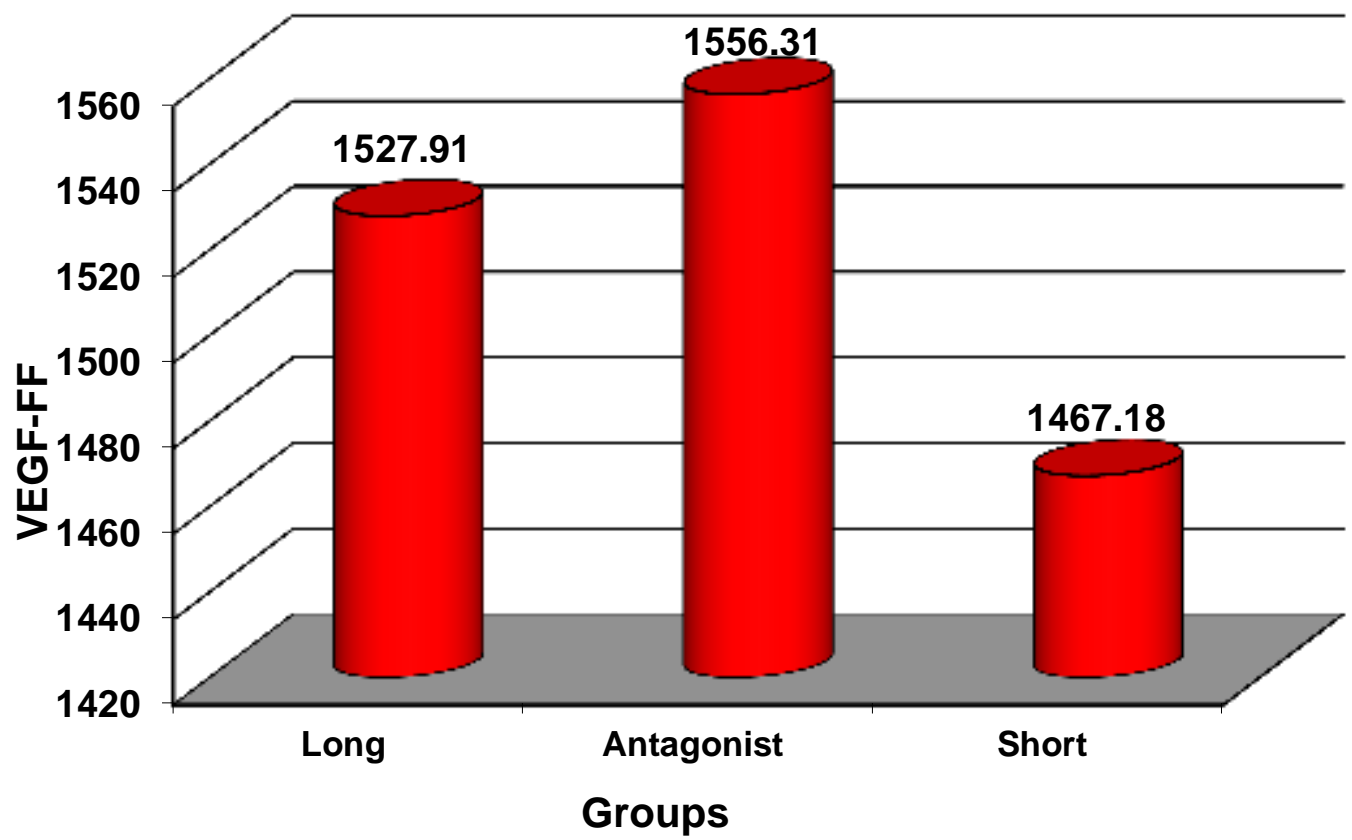

Figure 3. Comparison between different groups of PCOS in FF-VEGF

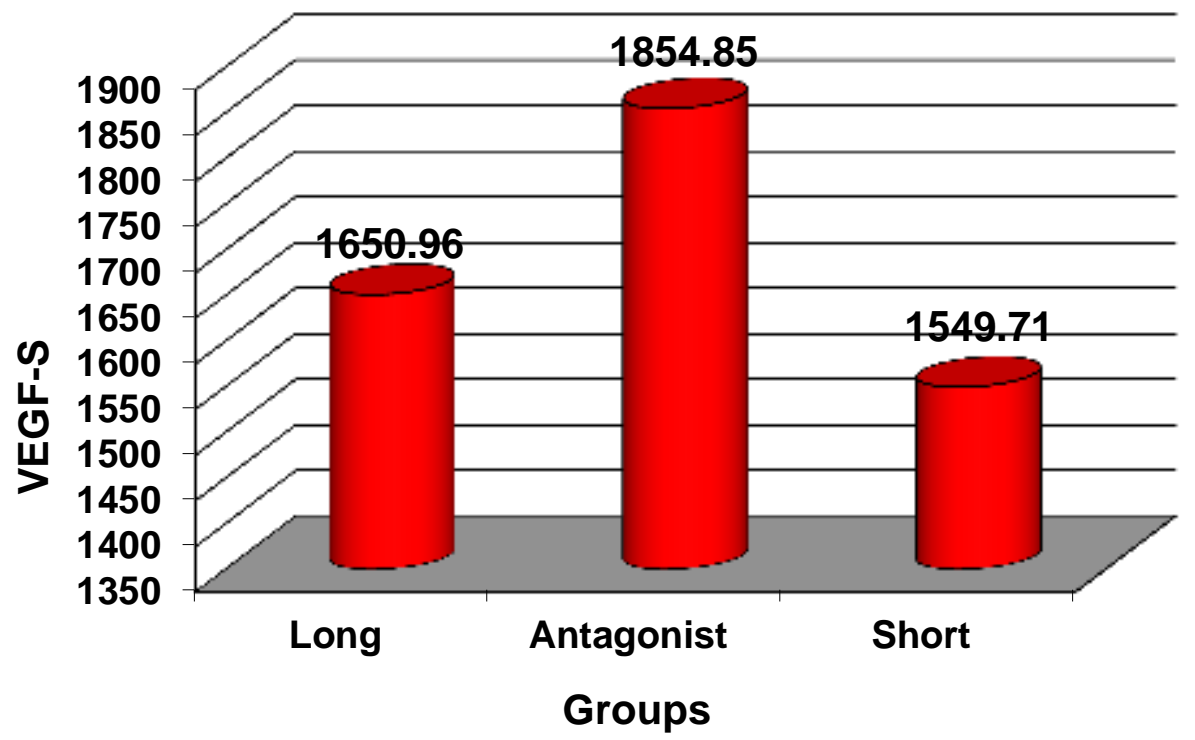

Figure 4. Comparison between different PCOS groups in S-VEGF 
Comparison of serum \& follicular fluid VEGF level between pregnant \&non pregnant PCOS patients:In our study the mean of serum and follicular VEGF levels at the day of oocyte retrieval \pm SE of pregnant PCOS patients shared in this study were $(1736.29 \pm 244.89$ and $1454.92 \pm 101.90)$ respectively. The mean of serum and follicular VEGF levels at the day of oocyte retrieval \pm SE of the non-pregnant PCOS group shared in this study were $(1661.20 \pm 109.34 \pm 110.17$ and $1538.95 \pm 101.97)$ respectively. The statistical analysis showed no significant difference $(\mathrm{P}>0.05)$ in level of serum VEGF between the two groups while shows significant difference $(\mathrm{P}<0.05)$ in level of follicular VEGF between pregnant \& non-pregnant PCOS patients. The mean of follicular VEGF levels was high in non-pregnant PCOS groups $(1538.95 \pm 101.97)$ in comparison with pregnant PCOS group $(1454.92 \pm 101.90)$ (Table 3).

Table 3:- Comparison of S \& FF- VEGF level between pregnant \&non pregnant PCOS patients:

\begin{tabular}{|c|c|c|c|}
\hline \multirow{2}{*}{$\begin{array}{c}\text { PCOS } \\
\text { Group }\end{array}$} & \multirow{2}{*}{ No. } & VEGF-S & Level of VEGF \\
\cline { 3 - 4 } & & Pg/ml & Pg/ml \\
\hline Pregnant & 13 & $1736.29 \pm 244.89$ & $1454.92 \pm 101.90$ \\
\hline Non pregnant & 42 & $1661.20 \pm 109.34$ & $1538.95 \pm 101.97$ \\
\hline T-Test & & $118.733 \mathrm{NS}$ & $76.224 *$ \\
\hline \multicolumn{2}{|c|}{$(\mathrm{P}<0.05)$, NS: Non-significant. } \\
\hline
\end{tabular}

\section{Discussion:-}

In current study shows no significant difference regarding serum and follicular fluid levels of VEGF between PCOS and non PCOS women. Our finding is in disagreement with Agrawal et al $\left(2002^{(4)}\right.$, Artini et al. (2006) ${ }^{(20)}$, Artini et al. (2009) ${ }^{(21)}$ and Mary et al (2016) ${ }^{(22)}$ who found elevated levels of VEGF in serum and follicular fluid of patients with PCOS undergoing IVF. The increased levels of VEGF explained by the finding that the release of VEGF by granulosa lutein cells is augmented by insulin ${ }^{(4,23)}$ and that luteinized granulose cells in women with PCOS are more sensitive to insulin ${ }^{(23)}$. Since many women with PCOS have hyperinsulinemia, hypersecretion of VEGF in women with PCOS may be due to hyperinsulinemia ${ }^{(24)}$, whereas Lee et al. ${ }^{(25)}$ explained the high levels of VEGF by the investigation of single nucleotide polymorphisms of VEGF gene, in 134 Korean women with PCOS and they concluded that the single nucleotide polymorphisms regulating the expression and activity of VEGF may be significantly associated with pathogenesis of PCOS. The non-significant result in this study may be due to small sample size or because of same BMI in both PCOS and non PCOS women $(28.68 \pm 1.06$ and $28.29 \pm 0.99) \mathrm{Kg} / \mathrm{m} 2$, respectively (Table 1). Mechanisms through which body mass affects reproduction that have been mentioned include deviations in endocrine and metabolic functions, probably because of altered insulin resistance and cause hyerinsulinemia that cause hypersecretion of VEGF, this may explain why we found no significant difference between two groups. Although our finding show statistically no significant difference in the S-VEGF level between PCOS and non-PCOS women but there is higher S-VEGF level in PCOS women(1678.95 \pm 100.50$)$ than in non PCOS women(1548.46 \pm 80.95 ) so significant result may be can obtained either by increase sample size or by use another method of statistics.

We found that serum VEGF levels were not significantly different between pregnant and non-pregnant PCOS groups. This is in agreement with Nikolettos et al. ${ }^{(26)}$ and Mary et al ${ }^{(22)}$ who found that serum VEGF levels were not significantly different between pregnant and non-pregnant PCOS group in women undergoing ICSI. On the other hand, we found lower FF VEGF levels in the group of pregnancy than those from the group of no pregnancy in PCOS women, and our finding is in agreement with Nikolettos et al. ${ }^{(26)}$ and Mary et al. ${ }^{(22)}$ who found that elevated concentrations of VEGF in follicular fluid correlate negatively with conception rates in assisted reproductive technologies. This negative impact of VEGF on pregnancy outcome may be explained by the finding of Barroso et al. (27) who found that follicular VEGF levels are negatively-correlated with embryo morphology. VEGF concentration in follicular fluid is said to be dependent on the quality and number of granulosa cells responding ${ }^{(28)}$. Greater granulosa cell numbers have been associated with more competent follicles and lower follicular fluid VEGF levels with more oocytes ${ }^{(29)}$ and better embryo quality ${ }^{(4,27)}$. Van Blerkom et al. ${ }^{(28)}$ demonstrated that VEGF is associated with follicular vascularization and oxygenation and those oocytes from severely hypoxic follicles ascertained by lower VEGF concentrations are associated with higher frequencies of abnormalities in chromosomal organization which can lead to disorders in developing embryos. This observation was supported by observations of Bokal and colleagues ${ }^{(11)}$ who reported that follicular maturation and oocyte quality are dependent on intrafollicular concentrations of VEGF and rennin, which affects the fertilization competence of an oocyte. 
The findings of the present study show that, in women with PCOS, GnRH Agonist and antagonists exert no different effect on the VEGF synthesis and discharge, which disagreed with a study done by Bokal and colleagues ${ }^{(12)}$ observed lower follicular VEGF concentrations in all aspirated follicles of women with PCOS treated by GnRH antagonists. Cunha-Filho et al., $2005^{(30)}$ and Asimakopoulos et al., $2006^{(31)}$ investigated the role of GnRH antagonists on VEGF production during minimally stimulated IVF cycles with monofollicular developments compared with the natural-cycle IVF. According to their results, follicular VEGF concentrations were also lower using GnRH antagonists, but the difference was not statistically significant. These results are not in complete agreement with the statistically lower follicular VEGF concentrations in the GnRH antagonist group but in agreement with our result. Contrary to the results of the present study and the above-mentioned studies ${ }^{(30,31)}$, Ferrari et al. (2006) ${ }^{(32)}$ found higher follicular VEGF concentrations with GnRH antagonists than with GnRH agonists. This opposite finding might be explained by the fact that in the study by Ferrari et al. the indications for IVF were various (tubal factor, unexplained infertility and endometriosis), whereas in this study the indications were PCOS and male factor infertility.

\section{References:-}

1. Byneil S and Charissa M. Polycystic Ovary Syndrome. Obstet. Gynecol. 2009;114: 936-49.

2. P. Peitsidis, R. AgrawalRole of vascular endothelial growth factor in women with PCO and PCOS: a systematic review. Reprod Biomed Online, 20 (2010), pp. 444-452.

3. Lim SS, Norman RJ, Davies MJ et al. The effect of obesity on polycystic ovary syndrome: a systematic review and meta-analysis. Obes Rev. 2013; 14: 95-109.

4. R. Agrawal, H. Jacobs, N. Payne, G. ConwayConcentration of vascular endothelial growth factor released by cultured human luteinized granulosa cells is higher in women with polycystic ovaries than in women with normal ovariesFertil. Steril., 78 (2002), pp. 1164-1169.

5. N. McClure, D.L. Healy, P.A. Rogers, et al.Vascular endothelial growth factor as a capillary permeability agent in ovarian hyperstimulation syndromeLancet, 344 (1994), pp. 235-2243.

6. García-Velasco and Pellicer, 2003J.A. García-Velasco, A. PellicerNew concepts in the understanding of the ovarian hyperstimulation syndromeCurr. Opin. Obstet. Gynecol., 15 (2003), pp. 251-256.

7. Sengupta J, Lalitkumar PG, Najwa AR, et al. Immunoneutralization of vascular endothelial growth factor inhibits pregnancy establishment in the rhesus monkey (Macaca mulatta).Reproduction. 2007; 133 (6):11991211.

8. Regnault TRH, Orbus RJ, De Vrijer B, et al. Placental expression of VEGF, PIGF and their receptors in a model of placental insufficiency-intrauterine growth restriction (PI-IUGR).Placenta. 2002; 23(2-3):132-144.

9. García-Velasco and Pellicer, 2003J.A. García-Velasco, A. PellicerNew concepts in the understanding of the ovarian hyperstimulation syndromeCurr. Opin. Obstet. Gynecol., 15 (2003), pp. 251-256.

10. F.A. Aleem, M. Predanic.Transvaginal colour Doppler determination of the ovarian and uterine blood flow characteristics in polycystic ovary diseaseFertil. Steril., 65 (1996), pp. 510-516.

11. E.V. Bokal, H.M. Vrtovec, I.V. Klun, et al. Prolonged HCG action affects angiogenic substances and improves follicular maturation, oocyte quality and fertilization competence in patients with polycystic ovarian syndrome Hum Reprod, 20 (2005), pp. 1562-1568.

12. E.V. Bokal, I.V. Klun, I. Verdenik. Follicular oestradiol and VEGF after GnRH antagonists or GnRH agonists in women with PCOS. Reprod Biomed Online. 2009; 18 (1):21-28.

13. Rotterdam ESHRE/ASRM-sponsored PCOS consensus workshop group: Revised 2003 consensus on diagnostic criteria and long-term health risks related to Polycystic ovary syndrome (PCOS), Hum Reprod 19 .2004; pp. 41-47, Fertile Steril.; 81: 19-25. 11.

14. Hugues JN, Cédrin-Durnerin I. Endocrine characteristics of ART Cycles. In : Gradner DK, Weissman A, Howles CM, Shoham Z ,editors. Textbook of Assisted Reproductive Technologies, Volume Two: Clinical Perspective.4th ed. London: Informa Health care; 2012. pp. 99-114.

15. Elder K, Dale B. In-Vitro Fertilization.3rded. New York: Cambridge University Press. 2011. pp.19-27.

16. Zegers-Hochschild F, Adamson GD, de Mouzon J, et al. The International Committee for Monitoring Assisted Reproductive Technology (ICMART) and the World Health Organization (WHO) Revised Glossary on ART. Terminology. 2009. Hum. Reprod. 2009; 24: 2683-7. 87.

17. Al-Inany H, and Aboulghar M. GnRH antagonist in assisted reproduction:a Cochrane review. Hum Reprod. $2002 ; 17: 874-885$.

18. Schats R, Huirne JAF. The use of GnRH agonists. In : Gradner DK, Weissman A, Howles CM, Shoham Z, editors. Textbook of Assisted Reproductive Technologies, Volume Two: Clinical Perspectives, 4th ed.London: Informa Health care. 2012. pp.115-23. 
19. SAS. 2012. Statistical Analysis System, User's Guide. Statistical. Version 9.1th ed. SAS. Inst. Inc. Cary. N.C. USA.

20. P.G. Artini, M. Monti, C. Matteucci, et al.Vascular endothelial growth factor and basic fibroblast growth factor in polycystic ovary syndrome during controlled -ovarian hyperstimulationGynecol Endocrinol, 22 (2006), pp. 465-470.

21. P.G. Artini, M. Ruggiero, M.R. Parisen Toldin, et al.Vascular endothelial growth factor and its soluble receptor in patients with polycystic ovary syndrome undergoing IVF. Hum Fertil (Camb), 12 (2009), pp. 40-44.

22. Mary K., Marwan Al-H., Faizeh Al-Q. Follicular fluid Vascular Endothelial Growth Factor (VEGF) could be a predictor for pregnancy outcome in normo-responders and polycystic ovary syndrome women undergoing IVF/ICSI treatment cycles. Middle East Fertility Society Journal 2016; 3(21): 52-56.

23. M.B. Stanek, S.M. Borman, T.A. Molskness, et al.Insulin and insulin-like growth factor stimulation of vascular endothelial growth factor production by luteinized granulosa cells: comparison between polycystic ovarian syndrome (PCOS) and non-PCOS women.J Clin Endocrinol Metab, 92 (2007), pp. 2726-2733.

24. P. Peitsidis, R. Agrawal Role of vascular endothelial growth factor in women with PCO and PCOS: a systematic review Reprod Biomed Online, 20 (2010), pp. 444-452.

25. E.J. Lee, B. Oh, J.Y. Lee, et al. Association study between single nucleotide polymorphisms in the VEGF gene and polycystic ovary syndrome.Fertil Steril, 89 (2008), pp. 1751-1759.

26. N. Nikolettos, B. Asimakopoulos, A. Efthimiadou, et al.Evaluation of leptin, interleukin-1 $\beta$, tumor necrosis factor- $\alpha$ and vascular endothelial growth factor in serum and follicular fluids of women undergoing controlled ovarian hyperstimulation as prognostic markers of ICSI outcome.In Vivo, 18 (2004), pp. 667-674.

27. G. Barroso, M. Barrionuevo, P. Rao, et al.Vascular endothelial growth factor, nitric oxide, and leptin follicular fluid levels correlate negatively with embryo quality in IVF patients.Fertil Steril, 72 (1999), pp. 1024-1072.

28. J. Van Blerkom, M. Antczak, R. Schrander.The developmental potential of the human oocyte is related to the dissolved oxygen content of follicular fluid: association with vascular endothelial growth factor levels and perifollicular blood flow characteristics Hum. Reprod., 12 (1997), pp. 1047-1055.

29. C.I. Friedman, D.B. Seifer, E.A. Kennard, et al.Elevated level of follicular fluid vascular endothelial growth factor is a marker of diminished pregnancy potential. Fertil. Steril., 70 (1998), pp. 836-839.

30. Cunha-Filho JS, Lemos N, Stein N et al. Vascular endothelial growth factor and inhibin A in follicular fluid of infertile patients who underwent in-vitro fertilization with a gonadotropin-releasing hormone antagonist. Fertility and Sterility.2005; 83, 902-907.

31. Asimakopoulos B, Nikolettos N, Nehls B et al. 2006 Gonadotropin-releasing hormone antagonists do not influence the secretion of steroid hormones but affect the secretion of vascular endothelial growth factor from human granulosa luteinized cell cultures. Fertility and Sterility 86, 636-641.

32. Ferrari B, Pezzuto A, Barusi L et al. Gonadotropin-releasing hormone antagonists increase follicular fluid insulin-like growth factor-I and vascular endothelial growth factor during ovarian stimulation cycles. Gynecological Endocrinology.2006; 22, 289-29. 